\title{
Special issues for older women with primary breast cancer
}

\author{
Ruth Mary Parks ${ }^{1}$ (D) Yasmin Jauhari² ${ }^{\text {iD }}$, Jenna Morgan³ ${ }^{3}$, Lynda Wyld ${ }^{3}$ (D) \& \\ Kwok-Leung Cheung*,1 iD \\ ${ }^{1}$ Nottingham Breast Cancer Research Centre, School of Medicine, University of Nottingham, Royal Derby Hospital \\ Centre, Derby, DE22 3DT, UK \\ ${ }^{2}$ Clinical Effective Unit, Royal College of Surgeons of England, London, WC2A 3PE, UK \\ ${ }^{3}$ Department of Oncology \& Metabolism, The Medical School, University of Sheffield, Sheffield, S10 2SJ, UK \\ *Author for correspondence: kl.cheung@nottingham.ac.uk
}

"The future of treatment for older women with primary breast cancer should aim to utilize tumor and geriatric factors to deliver personalized cancer care, including in patients with dementia."”

First draft submitted: 29 April 2020; Accepted for publication: 18 May 2020; Published online: 10 June 2020

Keywords: biological assessment • geriatric assessment • older women • primary breast cancer

It is estimated that the number of older women living with breast cancer will quadruple by 2040 [1]. Despite this, there is a lack of strong evidence and guidelines to support management decisions for this population.

This article has evolved from a conference session on this subject, held at the 2020 UK Interdisciplinary Breast Cancer Symposium (UKIBCS; West Midlands, UK, 27-28 January 2020) [2]. The UKIBCS is a biennial meeting for all those with an active interest in breast cancer research and treatment, hosted by the charity Breast Cancer Now (London, UK), in collaboration with eight other breast cancer focused specialist societies.

In this editorial, we discuss key issues in the management of breast cancer in older women, which include both tumor and patient factors. We also review current national initiatives to tackle these issues and summarize ongoing work in this field.

\section{Key perspectives}

Tumor factors

Emerging data suggest that the biology of breast cancer in older women is different to that in younger women, although overall research work is still lacking in this area [3].

The Nottingham group describes possibly the largest consecutive series of older women with primary breast cancer, with both clinical follow-up data and tumor samples available, documented in the literature. Between 1973 and 2010, 1758 women $\geq 70$ years of age with early stage (clinically $\leq 5 \mathrm{~cm}$ ) breast cancer without evidence of metastases at diagnosis presented to the unit.

Work in this cohort has shown there are differences in survival outcome based on the degree of ER positivity. In a subset of 1085 patients, those with tumors with ER H-score of $\geq 250$ (out of 300, high ER expression) were found to have equivalent breast-cancer-specific-survival (BCSS) regardless of primary surgery or primary endocrine therapy (PET), whereas for patients with tumors with an H-score $<250$, surgery produced better BCSS ( $p<0.001$ ) [4].

Another example is in those patients with HER2-positive tumors. A comparison of 130 younger ( $<70$ years) patients with 43 older patients identified as having HER2-positive tumors found no difference in BCSS, despite minimal use of chemotherapy in the older age group, compared with frequent use in the younger group [5]. These results were replicated in patients with triple negative breast cancer [6]. This suggests that, overall, older women express a less aggressive phenotype of breast cancer compared with their younger counterparts.

The Nottingham group has gone on to assess a panel of 25 biomarkers in 575 older women undergoing surgery [3]. Cluster analysis demonstrated a novel subtype - low ER luminal (with low ER expression but high expression of 
luminal cytokeratins), previously not seen in younger women. Its identification may explain differences in clinical outcome among older women and needs future investigations.

\section{Patient factors}

Apart from biological differences in the tumor, the older population has unique personal needs, which should be considered in treatment discussions. Comprehensive geriatric assessment (CGA) is a process used by healthcare professionals to assess an older person's physical health and function, mental health and social circumstances. Geriatric assessment is recommended by the International Society of Geriatric Oncology (Châtelaine, Switzerland) [7] but there is no consensus on how it should be implemented or the results interpreted.

Alongside the work described above, the Nottingham group has an ongoing multicenter international prospective study exploring the potential value of CGA in primary breast cancer patients. A cancer-specific validated CGA tool [8], as well as measures of quality of life (QoL) are used within 6 weeks of diagnosis and again at 6 months. Preliminary results show that increasing age, comorbidity, number of medications and slower 'timed-up-and-go' score are associated with higher likelihood of receipt of PET over surgery [9].

When exploring treatment decision making with patients, they felt breast cancer treatment was a 'trade off' between risks and benefits of treatment. Furthermore, older women may value QoL and retention of functional independence more highly than extending the length of life $[8,10]$.

\section{Current national initiatives}

\section{Bridging the Age Gap study}

The Age Gap study is a multicenter prospective cohort study aiming to optimize the management of older ( $>70$ years) women with breast cancer [11]. This is an extremely important piece of research due to the difficulty of randomization in this population, resulting in a paucity of randomized controlled trial data. The Age Gap study is collecting observational real-world data but then using propensity score matching to adjust for treatment allocation bias rather than randomization. The key question for the Age Gap study was to determine which subgroup of older women could be safely offered nonstandard care (i.e., PET for primary treatment and omission of chemotherapy as adjuvant treatment) based on their health, fitness, frailty and tumor characteristics. A key element of the study was to collect detailed QoL data on all patients in light of the importance of this to women in this age group.

The study is now complete and data analysis is ongoing. The study recruited over 3400 women over the age of 70 years from 57 centers in England and Wales. Preliminary results discussed here were presented at the UKIBCS and are yet to be published [2].

A comparison of 672 patients (matched for age, frailty and comorbidity) receiving either PET or surgery showed no difference in BCSS regardless of treatment. After treatment, patients in both groups saw an initial decrease in QoL from baseline; however, this recovered for patients receiving PET, but not for patients undergoing surgery by the 2-year mark. This suggests that there is a group of older women who will have no oncological benefit from surgery and in fact, surgery may be detrimental to their QoL.

The second phase of the study involved the development of a predictive tool to tailor treatment options according to breast cancer stage and biology and their age, fitness and frailty. A Decision Support Instrument (DESI) was developed to assist older women in making informed decisions about their preferred treatment [11,12]. A multicenter cluster randomized trial was then nested within the main Age Gap observational study in 46 of the 57 centers. These 46 centers were randomized to continue with normal decision-making practice or to have training and the use of the online DESI. The trial has now completed recruitment and randomized 670 patients to the intervention (use of the DESI) and 669 to standard care. Preliminary results show early trends toward improved QoL and identical BCSS in patients who use the DESI, compared with those treated by standard care. The online tool is now freely available to use [12].

\section{A view on dementia}

The National Audit of Breast Cancer in Older Patients is part of an effort to evaluate the quality of cancer care provided to older ( $>70$ years) women with breast cancer in England and Wales [13]. At the time of publication of the third annual report in 2019 [13], 147,162 older women diagnosed with breast cancer between 2014 and 2017 had been recruited from 130 centers. 
In the 2017 report, a series of case vignettes were used to gain perspective on patient factors that influence clinical treatment decisions. The results highlighted inconsistencies in primary treatment preferences of women with breast cancer and complex multimorbidities such as cognitive impairment and dementia [14].

Fewer women with dementia present with breast cancer at an early stage. In a large $(n=31,935)$ retrospective cohort study, Raji et al. [15] found that $28 \%$ of patients with breast cancer and dementia were diagnosed with stage I disease, compared with $50.7 \%$ of patients without dementia. Furthermore, it is reported that women with dementia are up to $40 \%$ less likely to have surgery [16] compared with women without dementia. The reasons for this are complex and multifactorial. For example, patients with dementia are more likely to have competing comorbidities which may affect their suitability for general anesthesia [17]. Cohort studies have demonstrated a higher rate of postoperative complications in patients with dementia and breast cancer [18], as well as long-term cognitive effects and compliance issues for adjuvant therapy [19], but studies of this kind are few. At last, the impact of dementia and breast cancer on a patient's life expectancy remains poorly understood.

Given the increasing global burden of dementia [20], it is proposed that the approach to a patient with dementia should be as any other comorbidity - recognition, optimization and management that involves shared decision making.

\section{Conclusion}

Both tumor and patient factors are different in the older compared with younger woman with breast cancer. The future of treatment for older women with primary breast cancer should aim to utilize tumor and geriatric factors to deliver personalized cancer care, including in patients with dementia. This could be supported using tumor-based prognostic and predictive tools, geriatric assessment and patient decision-making tools. Research and audits in this area provide important insights and should continue.

\section{Acknowledgments}

The views expressed are those of the authors and not necessarily those of the National Health Service, the NIHR or the Department of Health.

Financial \& competing interests disclosure

The Age Gap study represents independent research funded by the National Institute for Health Research (NIHR) under its Programme Grants for Applied Research Programme (grant reference number: RP-PG-1209-10071). The authors have no other relevant affiliations or financial involvement with any organization or entity with a financial interest in or financial conflict with the subject matter or materials discussed in the manuscript apart from those disclosed.

No writing assistance was utilized in the production of this manuscript.

\section{Open access}

This work is licensed under the Attribution-NonCommercial-NoDerivatives 4.0 Unported License. To view a copy of this license, visit http://creativecommons.org/licenses/by-nc-nd/4.0/

\section{References}

Papers of special note have been highlighted as: • of interest

1. World Health Organization. International agency for research on cancer (2019). iarc.f $\mathrm{r}$

2. Cheung KL, Ring A. Special issues for elderly patients session. Presented at: UK Interdisciplinary Breast Cancer Symposium 2020: Breast Cancer Research and Treatment. Birmingham, UK (27-28 January 2020).

- You can find all of the conference proceedings from the UK Interdisciplinary Breast Cancer Symposium 2020 here.

3. Syed BM, Green AR, Paish EC et al. Biology of primary breast cancer in older women treated by surgery: with correlation with long-term clinical outcome and comparison with their younger counterparts. Br. J. Cancer 108(5), 1042-1051 (2013).

- This is a summary of analysis of a panel of biological markers in a large cohort of older women with primary breast cancer. To the best of our knowledge, this cohort is the largest of its kind, for this type of research, worldwide.

4. Syed BM, Al-Khyatt W, Johnston SJ et al. Long-term clinical outcome of oestrogen receptor-positive operable primary breast cancer in older women: a large series from a single centre. Br. J. Cancer 104(9), 1393-1400 (2011).

5. Syed BM, Green AR, Ellis IO, Cheung KL. Human epidermal growth receptor-2 overexpressing early operable primary breast cancers in older ( $\geq 70$ years) women: biology and clinical outcome in comparison with younger (<70 years) patients. Ann. Oncol. 25(4), 837-842 (2014). 
6. Syed BM, Green AR, Nolan CC, Morgan DAL, Ellis IO, Cheung KL. Biological characteristics and clinical outcome of triple negative primary breast cancer in older women: comparison with their younger counterparts. PLoS ONE 9(7), e100573 (2014).

7. Wildiers $\mathrm{H}$, Heeren $\mathrm{P}$, Puts $\mathrm{M}$ et al. International society of geriatric oncology consensus on geriatric assessment in older patients with cancer. J. Clin. Oncol. 32(24), 2595-2603 (2014).

8. Hurria A, Gupta S, Zauderer M et al. Developing a cancer-specific geriatric assessment: a feasibility study. Cancer 104(9), 1998-2005 (2005).

9. Parks RM, Hall L, Tang SW et al. The potential value of comprehensive geriatric assessment in evaluating older women with primary operable breast cancer undergoing surgery or non-operative treatment: a pilot study. J. Geriatr. Oncol. 6(1), 46-51 (2015).

- This is a pilot study examining the use of a breast cancer specific comprehensive geriatric assessment, specifically in older women with primary breast cancer.

10. Zahit RA, Parks RM, Howard P, Blake H, Cheung KL. Baseline quality of life evaluation in older women with primary operable breast cancer and its relationship with different treatments. J. Clin. Oncol. 36(Suppl. 15), e12601 (2018).

11. The University of Sheffield. Bridging the age gap in breast cancer (2020). www.sheffield.ac.uk/medicine/research/research-themes/cancer/age-gap

- This is the main reference to the Bridging the Age Gap study and contains information on study design, analysis and relevant publication to date.

12. The University of Sheffield. Age gap decision tool (2020). https://agegap.shef.ac.uk/

13. National Audit of Breast Cancer in Older Patients. NABCOP: annual report 2019 (2019). www.nabcop.org.uk/reports/nabcop-2019-annual-report/

- This 2019 report from National Audit of Breast Cancer in Older Patients contains the most recent published findings from this nationwide audit.

14. National Audit of Breast Cancer in Older Patients. NABCOP: annual report 2017 (2017). www.nabcop.org.uk/reports/nabcop-2017-annual-report/

15. Raji MA, Kuo Y-F, Freeman JL, Goodwin JS. Effect of a dementia diagnosis on survival of older patients after a diagnosis of breast, colon, or prostate cancer: implications for cancer care. Arch. Intern. Med. 168(18), 2033-2040 (2008).

16. Gorin SS, Heck JE, Albert S, Hershman D. Treatment for breast cancer in patients with Alzheimer's disease. J. Am. Geriatr. Soc. 53(11), 1897-1904 (2005).

17. Prince M KM, Guerchet M, McCrone P et al. Dementia UK: update second edition report produced by KCL and the LSE for the Alzheimer's society (2014). http://eprints.Ise.ac.uk/59437/1/Dementia_UK_Second_edition_-_Overview.pdf

18. Hu CJ, Liao CC, Chang CC, Wu CH, Chen TL. Postoperative adverse outcomes in surgical patients with dementia: a retrospective cohort study. World J. Surg. 36(9), 2051-2058 (2012).

19. Phillips KA, Ribi K, Sun Z et al. Cognitive function in postmenopausal women receiving adjuvant letrozole or tamoxifen for breast cancer in the BIG 1-98 randomized trial. Breast 19(5), 388-395 (2010).

20. GBD 2016 Dementia Collaborators. Global, regional, and national burden of Alzheimer's disease and other dementias, 1990-2016: a systematic analysis for the global burden of disease study 2016. Lancet Neurol. 18(1), 88-106 (2019).

- This study is important in understanding the impact of dementia in the population on a worldwide scale and has significant findings. 